$\xi=-1$

\title{
Models, Methods and Tools of Optimizing Costs for Development of Clusterized Organizational Structures in Construction Industry
}

\author{
M. Mykytas ${ }^{1}$, S. Terenchuk²*, N. Zhuravska ${ }^{3}$, \\ ${ }^{1}$ Kyiv National University Of Construction And Architecture, Ukraine \\ ${ }^{2}$ Kyiv National University Of Construction And Architecture, Ukraine \\ ${ }^{3}$ Kyiv National University Of Construction And Architecture, Ukraine \\ *Corresponding Author E-Mail:Terenchuksa@Ukr.Net
}

\begin{abstract}
The mission of this research shall be studying the models and methods of evaluation of the expected costs to be used in economic efficiency optimization of clusterized organizational structures in construction industry. It comprises analysis of models and methods of simulation of the development of complex models under fuzzy conditions. It shows benefits and drawbacks of realizing the scenario approach based on econometric semi-linear dynamic equations. The optimization of costs has been proposed to be performed based on simulation modelling of various scenarios of development of clusterized organizational structures of construction branch. The results of simulation can be useful in feasibility demonstrations of the long-term plans and strategies of development on various stages of structures' life cycle. The mathematical basics and scheme of forming the minimum costs for providing the best strategy of development of clusterized organizational structure. The academic novelty of work consists in developing the rationale of application of fuzzy neuron boundaries. The responsibility for the development of fuzzy rules in order to form system fuzzy databases shall be vested in experts.
\end{abstract}

Keywords: clusterized organizational structure, economic and mathematical models, fuzziness, neuron network.

\section{Introduction}

Beginning in 2014 the integrated sustainable urban development [1] has become a focus area of European regional policy. Such policy has resulted in emergence of the new technical and organizational systems - clusters [2]. Clusterization has become one of the prevailing trends in economic and environmental modernization of locations in Ukraine.

The important role in forming and developing the clusterized organizational structures (COS) is played by system capacity for self-organization, emergence and synergy. The impact of systematic properties on cluster-forming mechanism is ambiguous while the scenarios of developing each formed structure remain multivariant throughout the entire life cycle [2]. The multivarience of development is related to the action of various superpositions of stochastic factors, the degree of impact of each of them as determined, in each separate case, by the condition of environment and properties of the system itself.

Under such circumstances, it is still actual to develop:

- reliable models and methods of evaluating the expected economic efficiency and possible economic implications of operation of clusterized organizational structures in the rationale of longterm plans and strategies of development [3, 4];

- decision-making maintenance systems capable of providing evidence-based support to management of development of clusterized organizational structures on various stages of their life cycle in fuzzy conditions [5].

Modern methods and tools of managing complex systems that are clusterized organizational structures of construction industry are based on forecasting the implications of a vast number of trajectories of probabilistic processes whereas implementing each of them in real conditions shall be possible as a result of impact of various superpositions of stochastic factors.

However, the fuzziness as related with future changes in external and internal environment and improvement of applied software tools provides for the growth in demand for intellectual systems with fuzzy logic capable of solving multicriterial tasks under conditions of ambiguity and risks.

\section{Analysis of Models and Modeling Techniques Complex Systems' Development}

In order to forecast the future values of indicators describing economic efficiency of system operation the various methods of data processing can be used. The approach as based on assumption about reflecting of mathematical image of process undergoing in the real system by time-series is the best for simulating the dynamic processes.

\subsection{Time-Series Analysis Objectivesm}

The purpose of analysis of time-series is obtaining useful information for developing mathematical model of the process from some sequence of data reflecting the real process in the system. The primary task to be solved in simulating trajectories of random processes is determining the phase-space. To this end, it is necessary to determine the essential factors expedient to be used as phase-value. The process image is built on phase space whose 
coordinates are independent variables describing state of dynamic system. Other important task of time-series analysis upon which the quality of model is substantially reliant consists in separating the data structure from random fluctuations and noises. In solving this task, the statistical analysis of time-series is performed.

\subsection{Statistical Analysis of Time-Series}

For a long time the centerpiece in statistical analysis of time-series has been taken by single-factor linear models ARMA, as defined by components characterizing the auto-regression and moving average [6]:

$$
x_{t}=a_{0}+\sum_{i=1}^{p} a_{i} x_{t-i}+\sum_{i=1}^{q} b_{i} \varepsilon_{t-i}+\varepsilon_{t}
$$

where, $x_{t}$ - character of certain value observation result; $p$ - auto regression order; $q$ - moving average order; $a_{0}, a_{i}, b_{i}$ - parameters of model; $t=1,2, T$ - time intervals; $\varepsilon_{t}$ - noise.

The multifactor models are used in cases of connection of target variable with various time series [6]:

$$
x_{t}=a_{0}+\sum_{k=1}^{N} \sum_{i=1}^{p(k)} a_{i}^{(k)} x_{t-\tau_{i}}+\varepsilon_{t}
$$

where, $k$ - is number of time-series.

The simplicity of linear models allows forecasting with the use of standard packages of numeric methods, however, the specified models quickly lose relevance and require constant re-evaluation of model parameters in modelling non-linear processes. Therefore, the next stage of tool development for the time-series analysis has become the development of tools capable of factoring in the nonlinear nature of processes flowing in real systems. The STAR- and TAG- models have been proposed representing linear combination of models with coefficients that are continuous time functions. With artificial neuron network development the new opportunities of modelling the non-linear economic processes have emerged.

\subsection{Neuron Network-Based Models}

The neuron networks can be considered as generalization of conventional approaches to time-series analysis providing additional opportunities in simulating linear phenomena and recognizing chaotic processes.

Any relation of the type [7]:

$x_{t}=f\left(x_{t-1}, x_{t-1}, \ldots, x_{t-p}\right)+\varepsilon_{t}$

with continuous non-linear function can be renewed by multilayer network. It means that all models described in previous paragraphs can be implemented with neuron networks. In lieu of reflecting the surface as created by data with one hyperplane (AR), several hyperplanes (TAG) or several smoothly-connected hyperplanes (STAR) in phase space, the neuron network is capable of making random non-linear reflection.

So far, a great number of neuron network implementing software packages has been developed. The most important are such programs-simulators of neuron networks as: Nestor, Cascade Correlation, Neudisk, Mimenice, NuWeb, Brain, Data, Neuralworks Professional II / Plus, Brain Maker, HNet, Explorer, Explonet 3000, Neurosolutions, Propagator, Matlab Toolbox.

Additionally, there are other simulators which are freely distributed through university servers, for example SNNS (Stuttgart) or Nevada QuickPropagation.

However, the research in the field of simulation by means of artificial neuron networks has continued so far without any standard methods having been worked out. The problems and complications occur in case of overlaying of great number of stochastic dynamic factors. Under such circumstances only heuristical approach is possible.

\section{Designing the decision-making support sys- tem in cost-optimization task}

It is recommended to designate profitability of structure operation within the specified period [4] as a target function in the decisionmaking support system of optimization of costs for the development of clusterized organizational structure of construction industry [4]:

$\varphi=\frac{P(t+\Delta t)}{W}$

where, $\varphi$ - profitability; $P$ - profit for the specified period of time $t+\Delta t ; W$ - corresponding costs.

\subsection{Economic and Mathematic Rationale of the Task}

The profitability index provides opportunity to assess the expected economic efficiency of operation of clusterized organizational structure as a whole and meets all requirements as put to optimization criteria: determined, systematic, sensitive.

The optimal value will be the biggest of alternative values of target function subject to conditions: $P n \mathrm{p} \rightarrow \max ; W n p \rightarrow \min$ and in consideration of other restrictions.

The integrated key indicator [8] shall be considered expenses $\left(W_{i j}\right)$ for the maintenance of specified modes of functioning of clusterized organizational structure and adapting the structure to the changes of external environment for the specified period of time. In particular: expenses for pre-project preparation $-W_{l j}$; expenses for projecting $-W_{2 j}$; construction expenses $-W_{3 j}$; maintenance expenses $-W_{4 j}, i-$ index that determines life cycle stage of clusterized structure; $j-$ structure identifier (Fig. 1)

The scheme on Fig.1 represents formation of the model for evaluating criteria of optimizing the economic efficiency of operation of clusterized organizational structures in compliance with econometric semi-linear equations [4]:

$$
\begin{aligned}
& \varphi_{t+1}^{o n m}=\sum_{1 \leq i \leq I} \alpha_{i j} W_{i j}^{o n m}(t) \\
& W_{i j}(t)=\sum_{\substack{1 \leq j \leq J \\
1 \leq k \leq K \\
1 \leq l \leq L}} \beta_{j k} Y_{j k}\left(x_{m n, t-l}, \varepsilon\right),
\end{aligned}
$$

where: $\varphi_{t+1}^{o n m}$ - expected value of target function; $W_{i j}-j$-key indicator; $t$ - time; $Y_{j k}$ - function reflecting impact of $k$-factor on key indicator; $x_{m n}$ - explanatory factors $(1 \leq m \leq M, 1 \leq n \leq N) ; l-$ order of delay; $\alpha \mathrm{ij}, \beta \mathrm{jk}$ - adaptation parameters.

Identification of model parameters consists of two stages. On the first stage there is authentication of equations with variables (5) with indicators of operation of clusterized structure.

Authentication of variables and determination of phase space are followed by evidence-based and convenient for forecasting hypotheses reflecting dependencies of costs of each type of operation of structural units of clusterized organizational structures of impact factors. 


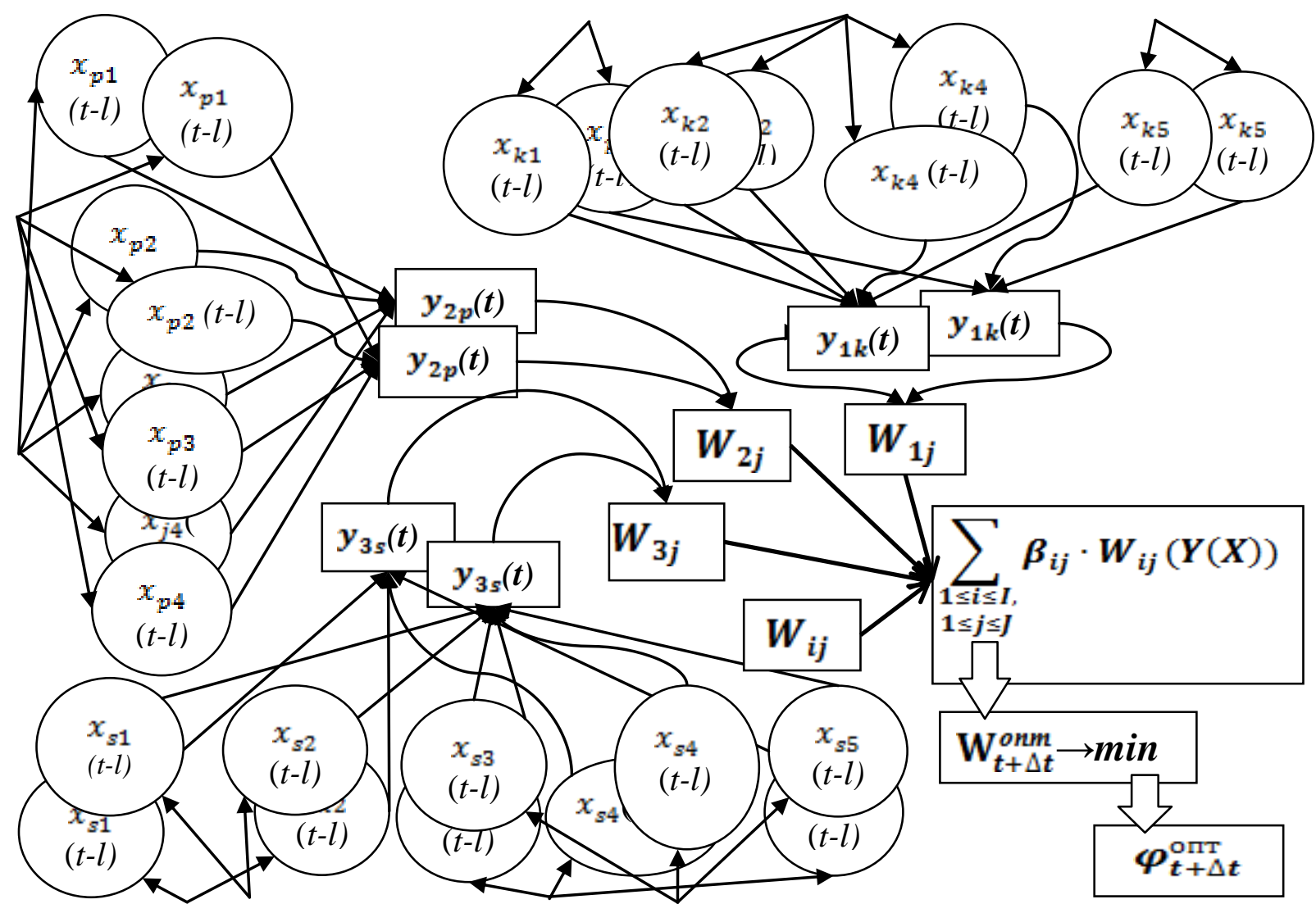

Fig. 1: Generalized diagram of forming model for simulation experiments:

$\varphi$ - target function; $W$ - costs for expected achievement of value of target function; $i$ - type of activity; $j$ - code of object;

$\beta_{i j}$ - assessment of parameters of impact; $y$ - resource; $k, p$, $s$-types of resource; $x$ - explanatory factors; $l$-order of delay

For example:

\section{INFRASTRUCTURE FACTORS OF IMPACT $\left(Y_{2 p}\right)$}

Expenses for ensuring reliability and safety of construction facilities $\left(y_{22}\right)$

$$
\begin{aligned}
& \text { Projecting }\left(x_{21}\right) \\
& \text { Construction }\left(x_{22}\right) \\
& \text { Operation }\left(x_{23}\right)
\end{aligned}
$$

Engineering system optimization costs $\left(y_{23}\right)$

Projecting $\left(x_{31}\right)$

Construction $\left(x_{32}\right)$

Operation $\left(x_{33}\right)$

Expenses for provision of transportation connection $\left(y_{24}\right)$

Projecting $\left(x_{41}\right)$

Conducting $\left(x_{42}\right)$

Operation $\left(x_{43}\right)$

\section{IMPACT OF TECHNOLOGICAL FACTORS $\left(Y_{3 s}\right)$}

Costs for intellectual designs $\left(y_{31}\right)$

Costs for software $\left(y_{32}\right)$

Costs for innovations $\left(y_{33}\right)$

Projecting $\left(x_{31}\right)$

Conducting $\left(x_{32}\right)$

Operation $\left(x_{33}\right)$

On the second stage, there is adjustment of model parameters and correlation and regression analysis of the degree if impact of guided variables of the model (5), which essentially impact the result- ing indicator of operation of clusterized organizational structures without correlation therewith

It must be noted that in real complex systems such as clusterized organizational structures, the independence condition, as a rule, is not performed. This is one of the obstacles to working out adequate econometric models but in modelling real processes 'pure' data are rare. In addition, real data contain noises and are unevenly distributed. Artificial neuron networks have been developed just for such cases [9-11].

For example:

1. Radial basis functions (RFB-networks) have been designed to solve classification problems based on renewal of mixtures of distributions and suit conditions under which it is difficult to determine the degree of impact of each factor of environment, however, the RBF-network application requires organizing vast data sampling for teaching. However, accumulation of sufficient quantity of typical examples for network teaching is considerably complicated by uniqueness if each clusterized organizational structure of construction industry [3, 10].

2. Kohonen network (SOM) is designed for solving tasks of clusterized analysis without teacher however, in cases of linearly undivided spaces of input data the quality of classifier is greatly reduced and it is not always possible to build the linearly divided multitude of signs of economic efficiency indicators of complex system of construction industry [12].

\subsection{Designing System of Estimating Costs for the De- velopment of Clusterized Organizational Structure}

Justifying the long-term plans and electing the best strategy of development on various levels and stages of structures of construction industry envisages assessment of expenses for implementation of various strategies. To this end various decisionmaking maintenance systems are developed. The operation of 


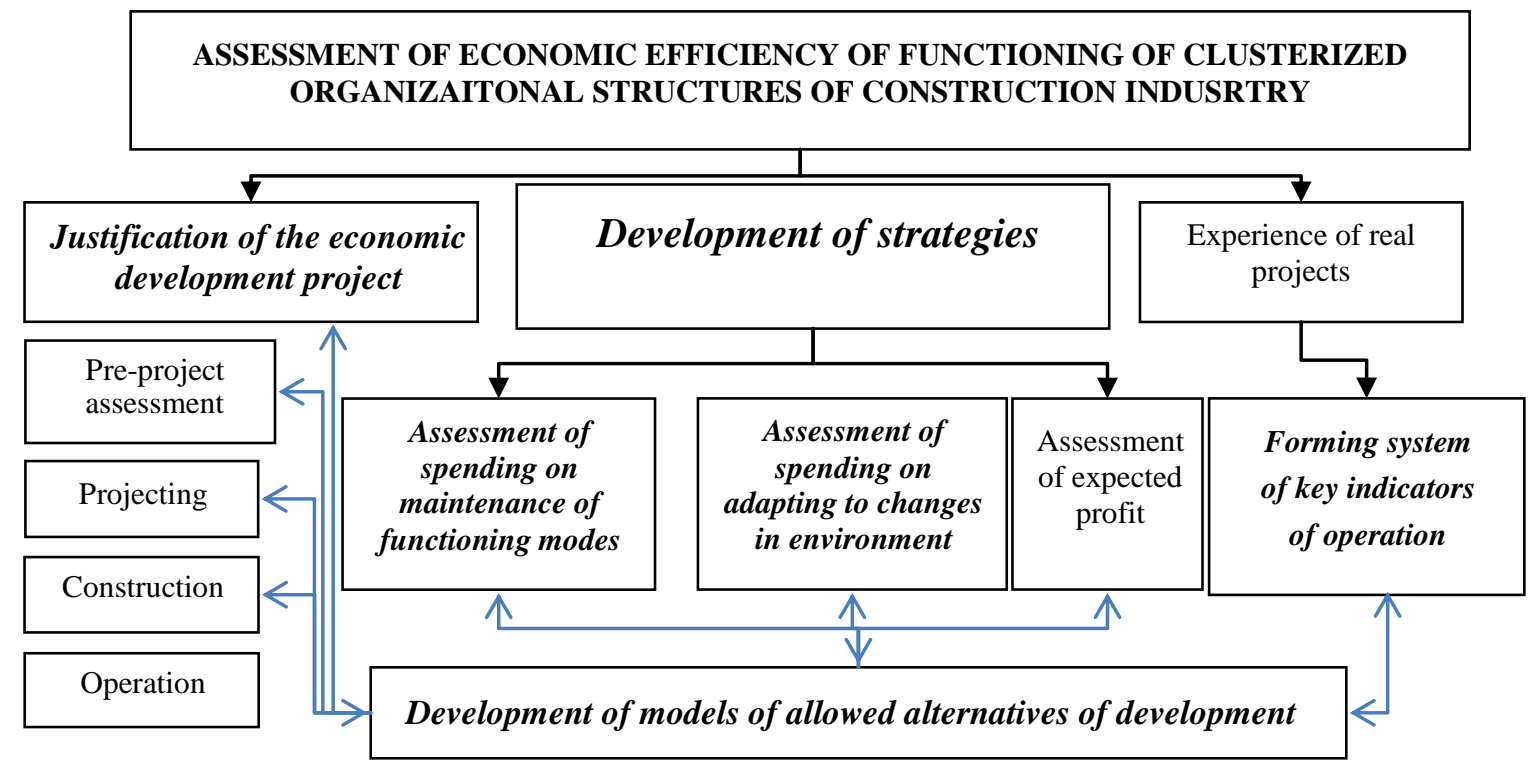

these systems is based on various models and methods of manaing complex systems (Fig.2).

Fig. 3 shows model of intelligent cost estimate system to be integrated into the process of the development of clusterized organizational structures.

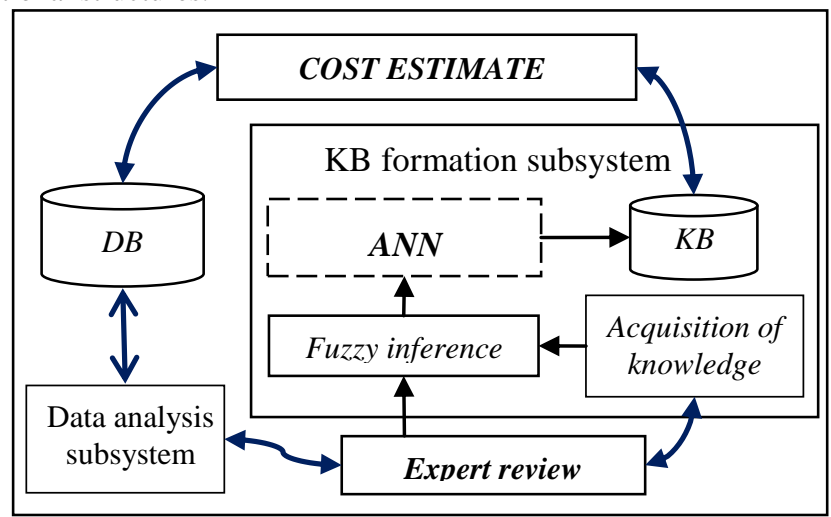

Fig. 3: Cost estimate assessment model

Designing estimate models is based on expert forming the knowledge that describe application and fill the knowledge base. Based on available knowledge and defined target function the system's metaprocedures generate and perform problem-solving task as well as form system logic explanation in accordance with its internal model [11]. Therefore, the intellectual system presents the informational system using the database and system of programs of its processing.

Cost estimate system consists of knowledge base (KB), database (DB), knowledge acquisition system, 'fuzzy inference' module, data analysis subsystem and provides for application of artificial neuron network (ANN).

Database (DB) contains economic and mathematical models and models required for time-series analysis, reference information necessary for conduct of simulated experiment with the aim of assessing the expected expenses for implementation of various strategies of development of clusterized organizational structures in various geographical, environmental, social and political conditions.

The fuzzy inference system includes [12]:

- set of fuzzy linguistic variables, with the help of which experts describe the type of defects and the state of the structures;

- rules on fuzzy variables;

- inputs and outputs of the system.

The intellectual system is the system that is based on knowledge and used for decision-making support and acquisition of the new knowledge based on knowledge engineering system. It is the knowledge engineering system defines methods of presenting and acquiring knowledge as well as architecture of expert systems for the account of unique organization of database and data interpreter guiding scheme (Fig. 4).

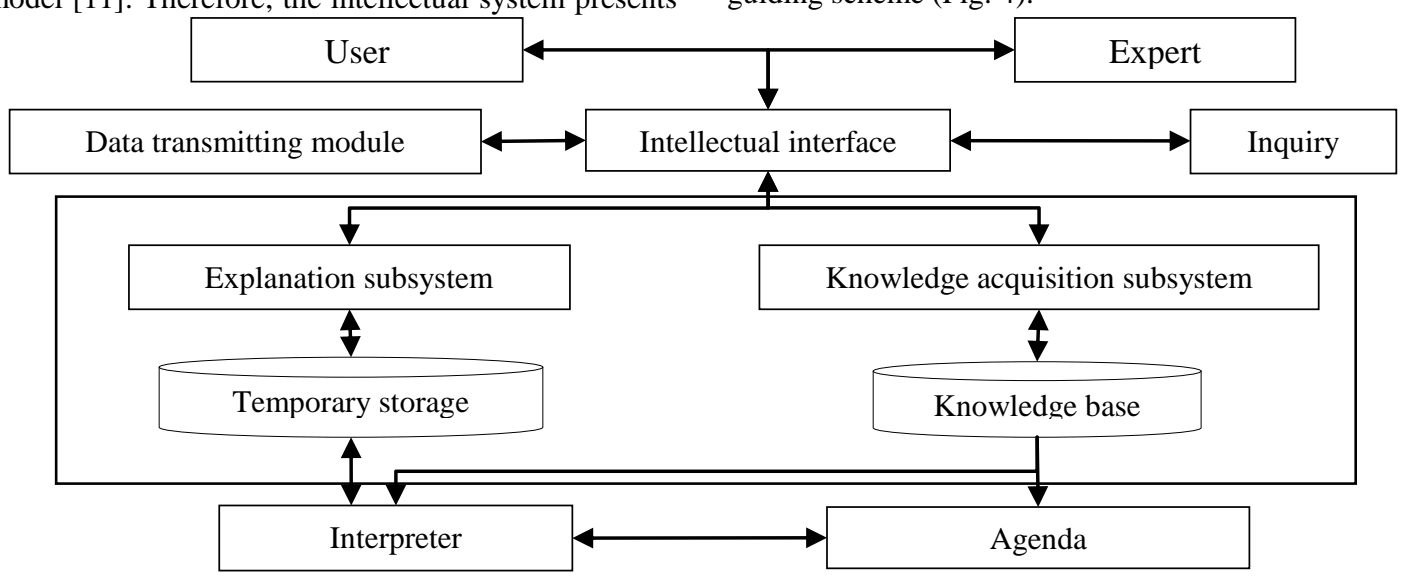

Fig. 4: Knowledge engineering system

In the context of study, the information tool contains all links between all variables of object and allows:

- calculating values of certain variables through the others;
- solving both direct and inverse problems;

- forecasting descriptions and properties of structures which have not yet been studied; 
- forecasting process parameters to obtain clusterized organizational structure with prescribed properties.

Knowledge base contains rules that have been elaborated by experts based on processing of the results of simulation experiments as conducted in order to forecast a multitude of trajectories of probabilistic processes the implementation of each of them being possible as a result of impact of different superpositions of stochastic factor. The data about trajectories of implemented processes and conditions of their implementations have been stored here as well.

The analysis and processing of statistical data as obtained in the course of observations is one of the main ways of acquiring knowledge about various changes in characteristics of system as a result of impact of various factors. However, every clusterized organizational structure of construction industry is unique and inert while modern outward conditions are unique and quickly change. It means that, as a rule, it is impossible to sample volume sufficient for network teaching from real data. In addition, a range of other problems emerge related with restricted access to internal information of structural units of cluster and processing of payloads in the form in which they have been obtained during observation.

In similar cases, forming of fuzzy knowledge base of cost estimate system is proposed to be made pursuant to analysis of computational experiments and to perform teaching of system based on simulation experiment data and multitude of rules representing formalized expert knowledge.

Module of 'knowledge acquisition' is designed for generating test tasks forming the teaching sample of artificial network.

The results of computational experiments represent a multitude of trajectories of random process which can be implemented in real conditions under impact of different superpositions of stochastic dynamic factors of external environment. The degree of impact of various factors and superpositions in each separate case is determined by experts.

The fuzzy database is a total of fuzzy rules in the following form [13]:

if <conditions of rule> then <conclusion of rule>,

determining the interrelation between inputs and outputs of study system.

If in this study the <original characteristic of cost estimate system is integral value of costs for development of clusterized organizational structure of construction industry>, then $<$ mathematical expectation of random process will determine costs for supporting the specified conditions of functioning of clusterized organizational structure> and <process dispersion reflects costs for adjusting the clusterized structure to random changes of environment> (fig. 2).

The acquired knowledge shall be stored until the period of forecasting is over. Based on the specified data the conclusions about reliability of forecasting and validity or falseness of rules (6), will be considered in further system teaching.

The 'fuzzy inference' module functions as 'white box' with reliability of operation ensured under conditions of uncertainty by human expert who can give formalized rule (6) based on analysis of results of simulation experiments.

The necessity for artificial generation of teaching sample is due to the fact that neural network forecasting is the most efficient method only in those cases when numerical series which are of non-linear nature or contain chaotic component have sufficiently representative 'history'.

\section{Conclusion}

The extensive use of artificial neuron networks for forecasting at the financial markets, concurrently with successful results, has revealed the unacceptable errors of forecasting. In particular: neuron networks do not perform satisfactory analysis of principally new situations due to insufficient representativity of the 'history of a series

Theory development toward improving neuron network based models enables to determine nature and interrelations of hidden and implied economic processes. Consideration of disclosed laws essentially impacts the reliability of long-term forecasting which allows to take timely actions in order to preserve or adapt the properties of economic systems to varying conditions of contemporary external environment and, thus, provide opportunity to reduce expenses for implementing the designated development strategy.

\section{References}

[1] Palahniuk Y.V. 'Transforming the financial instruments of cooperation between Ukraine and EU', Proceedings of the Institute of Legislation of Verkhovna Rada of Ukraine, \# 1, (2014), p. 99105.

[2] Murzin A., Anopchenko T. «Economic-Mathematical Modeling of Social and Environmental Risks Management of Projects of Urbanized Territories Development», Asian Social Science, 10(15), (2014), P. 249-254.

[3] Kostenko O.V. 'Defining the notion of 'cluster' from positions of systematic approach in economics', International scientific journal 'Innovative science', \# 9, (2015), p. 165-169.

[4] Shinkareva L., Plahov A. «Not formalized methods of the analysis of risk of the investment project», Economic and humanitarian sciences, 4(219), (2010), P. 8-11.

[5] Nasirzadeh F., Khanzadi M., Rezaie M. «Dynamic modeling of the quantitative risk allocation in construction projects», International Journal of Project Management, 32(3), (2014), P. 442-451.

[6] Vass H. U., Timmer J., Kurths J. «Nonlinear dynamical systems identification and indirect measurements», Int. J Bif. Chaos, 14, (2004), P. 1903-1933.

[7] McNelis, Paul D. «Neural network in finance: gaining predictive edge in the market», Elsevier Academic Press, (2005), 243p.

[8] Che Ibrahim C. K. I., Costello S. B., \& Wilkinson S. «Key indicators influencing the management of team integration in construction projects», International Journal of Managing Projects in Business, 8(2), (2015), P. 300-323.

[9] M S Sánchez, H Swierenga, L A Sarabia, E Derks, L Buydens. «Performance of multi-layer feedforward and radial base function neural networks in classification and modeling», Chemometrics and Intelligent Laboratory Systems, 2 (199606), P. 101-119.

[10] Tomaz Berlec, Alojzij Sluga, Edvard Govekar, et al. «Hybrid selforganization based facility layout planning», Strojnis ki vestnik, 60(12), (2014), P. 789-796.

[11] Blum, C., Puchinger J., Raid, J.R., Roli A. «Hybrid metaheuristics in combinatorial optimization: A survey», Applied Soft Computing, 11(6), (2011), P. 4135-4151.

[12] Hammah, R. «Fuzzy cluster algorithm for the automatic identification of joint sets», International Journal of Rock Mechanics and Mining Science, 35(7), (2010), P. 889-905.

[13] Terenchuk S., Yeremenko B., Kartavykh S., Nasikovsky O. «Application of fuzzy mathematics methods to processing geometric parameters of degradation of building structures», Eureka: physics and engineering», 1, (2018), P.56-62. 